\title{
Myocardial Threshold in Patients with Artificial Pacemakers*
}

\author{
Thomas A. Preston, B.s.e.e., m.D., $†$ Richakd D. Judge, b.s.e.e., M.d., \\ Benedict R. Lucchesi, PH.D., M.D. and David L. Bowers, B.s.e.E.
}

Ann Arbor, Michigan

$\mathrm{T}$ His report has a threefold purpose: (1) to describe a simple, clinical method of estimating myocardial threshold in patients with artificial pacemakers, (2) to verify the importance of critically high threshold as a significant cause of "pacemaker failure," and (3) to suggest for this type of failure a nonsurgical method of restoring myocardial responsiveness by administration of glucocorticoid and sympathomimetic drugs.

Although threshold problems have been previously reported in conjunction with at least three commercially available implantable pacemakers, ${ }^{1-3}$ the biologic consequences of prolonged artificial pacing have not been systematically investigated to date. Several reports have emphasized the technologic causes for pacemaker failure, ${ }^{4-7}$ and considerable effort has been expended toward the development of improved lcads, better pulse generators and longer battery life. These latter considerations are fundamentally engineering problems to which the physician directly contributes very little, except as he serves as an evaluator and advisor.

In calling particular attention to the biologic basis for pacemaker failure, referred to subsequently as exit block, we would like to emphasize the need for (1) ways of serially evaluating paccmaker function following implantation and (2) precise diagnosis of the cause of failure when it occurs unexpectedly. We feel the method here described holds great promise as means of meeting both of these needs.

\section{Materials and Methods}

In developing a means of quantitating the myocardial threshold level, we took advantage of the features incorporated into the design of our pacemaker (General Electric Co.), which allowed variation in the rate by an externally applied, inductioncoupled, control unit. By a similar method shown in Figurc 1, a thrcshold analyzer has been constructed which prevents the discharge of the output condenser of the implanted unit by introducing a high frequency (2,000 c.p.s.), low energy ( 1.45 microjoules) signal. The amplitude of each subsequent pacemaker stimulus is then inversely proportional to the number of suppression pulses introduced. By this means the rate of the implanted pacemaker can then be varied from 60 to 120 pulses per minute and the output energy per pulse can be suppressed to 2.5 per cent of normal. The technical details of this instrument will be reported elsewhere and are beyond the scope of this presentation.

Operation of the threshold analyzer can be performed by one physician. The electrocardiogram is observed continuously on a separate cardiac monitor. The first step is to place the induction coil directly over the implanted pacemaker. When sufficient inductive coupling is present, as registered by a simple galvanometer incorporated into the instrument, the analyzer is turned on and allowed to capture control of the rate of the implanted unit. The rate is then adjusted as desired, and the output signal of the implanted pacemaker is displayed on a Tektronix 502A oscilloscope with a 10 msec., synchronized sweep. This allows continuous monitoring of exact rate and pulse width. ${ }^{8}$ A simple electronic counter can also be used to display these two parameters. Finally, through one control dial, the energy delivered by the implanted unit can be slowly reduced. Threshold is

\footnotetext{
* From the Departments of Internal Medicine and Pharmacology, University of Michigan Medical Center, Ann Arbor, Mich. This investigation was supported in part by Public Health Service Research Grant 5M01-FR-42-05 from the Division of Research Facilities and Resources. Additional support was also received from grants awarded by the Horace H. Rackham School of Graduate Studies, University of Michigan Medical School, and the Michigan Heart Association.

$\dagger$ Winner of Second Place and Honorable Mention in the Young Investigators' Awards competition, February 5, 1966, at the Annual Meeting of the American College of Cardiology in Chicago.

$\ddagger$ Present address: Milwaukee, Wis.
} 


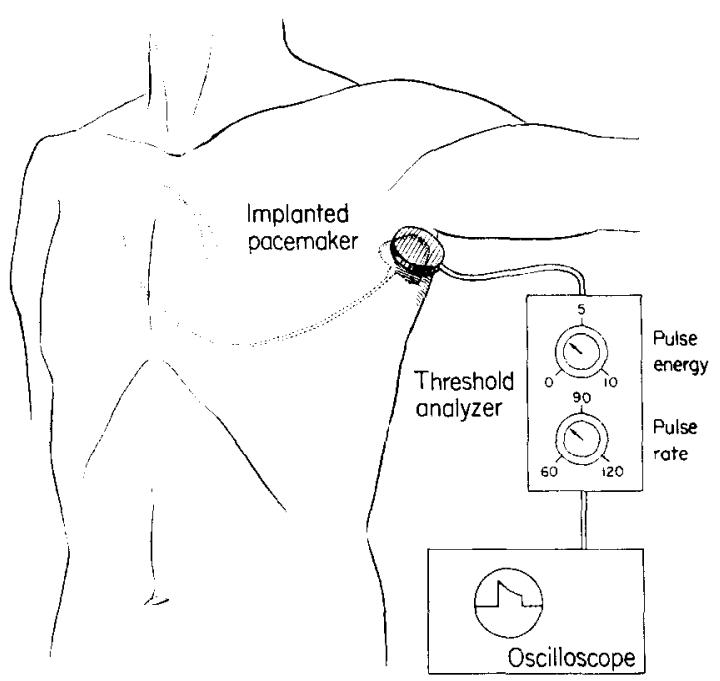

Fig. 1. Threshold analyzer. The instrument measures $15 \mathrm{~cm} . \times 20 \mathrm{~cm}$. $\times 15 \mathrm{~cm}$., is portable, and runs on two 6-volt mercury batteries. The coil at the end of the cable is placed directly over the implanted pacemaker. Garments need not be removed. Signals are relayed to an oscilloscope. The electrocardiogram is monitored continuously while the instrument is being used.

determined from the cardiac monitor as the point at whick response to the observed pacemaker stimulus no longer occurs. The pulse width is then quickly measured, and pacing is immediately restored.

This analyzer was first used on a laboratory model with a simulated biologic load of $300 \mathrm{ohms}$ and 47 microfarads. Direct measurements of current and voltage were then made. It was found that with this pacemaker circuit any given percentage decrease in output voltage results in a corresponding per cent decrease in pulse width. ${ }^{8,9}$ This is not a linear relationship, but is exactly the same for all pacemakers of this type, and does not vary with changes in interelectrode impedance within the biologic range. This means that when the threshold analyzer is used to reduce the pacemaker energy per pulse, for any per cent decrease in pulse width there is a corresponding per cent decrease in output voltage which can be determined from a calibration curve for the pacemaker. The same calibration curve is valid for all pacemakers of this type. Since encrgy is equal to the square of the voltage, divided by resistance, and resistance does not change as the voltage is decreased, the per cent energy in the reduced energy state is therefore equal to the square of the per cent of output voltage. Thus, for any reduction of pacemaker energy, the energy per pulse can be calculated as a per cent of available energy by first measuring pacemaker pulse width without reduction of energy, and then determining the pulse width at threshold. Without making direct measurements from the pacemaker wires, there is no way to determine absolute pacemaker energy, but any externally reduced or suppressed pacemaker energy can be calculated as a per cent of normally available pacemaker energy by this method.

A total of 50 threshold measurements zeere then made on 6 dogs with implanted pacemakers. To show that the threshold analyzer does not itself alter threshold, in $1 \mathrm{dog}$ threshold was measured by two different methods. The implanted myocardial electrodes were exposed, and threshold was determined by suppression with the threshold analyzer. Threshold energy was calculated from direct voltage and current measurements. Threshold was then determined by reducing the pacemaker energy supplied to the myocardial electrodes by mcans of resistances placed in series and in parallel with the myocardial electrodes. Threshold energy by the second method was also calculated by direct voltage and current measurements, and found to be exactly the same as that determined by the threshold analyzer, therefore demonstrating that the threshold analyzer itself does not alter threshold.

The threshold analyzer was first applied to a patient whose myocardial electrodes were exposed for direct measurements. Threshold energy was determined by the pulse width method described above, and simultaneously by measurements of direct current and voltage from the exposed electrodes. Calculation of threshold energy by these two methods varied by 1 per cent, which is within the limit of error for the pulse wid th method.

A total of 714 threshold measurements were then made externally on a series of 28 patients, by using the threshold analyzer as already described. In each case, threshold energy was calculated as per cent of total energy available. Figure 2 shows gradual reduction of pacemaker energy until the pulse energy is below that required for stimulation and asystole results. For purposes of standardization, we have defined threshold as that energy level at or below which at least one out of every ten pacemaker pulses fails to effect myocardial stimulation. For practical reasons, we attain threshold by slowly reducing pacemaker energy until one or more stimuli out of every ten fails to excite the heart. Threshold in all cases is reported as a per cent of available pacemaker energy.

Eighteen clinical cases of exit block were identified from 128 implantations over a four year perind. The criteria used for exit block were (1) rapidly increasing interelectrode impendance and threshold, (2) measurement of pulse width and rate to show that the pacemaker is functioning normally, and (3) clectrocardiographic demonstration of pacemaker stimuli without concurrent QRS complexes. The method of diagnosis of exit block by rate and pulse width analysis has already been reported in detail. ${ }^{8}$ Six surgically removed pacemakers were subjected to extensive technical evaluation and were found to be in perfect working order. Three cases of exit block have now been diagnosed and followed by accurate threshold measurements. In the other 15 cases crude measurements of threshold were possible by use of an external rate control, which causes decreased energy output as the rate is increased. ${ }^{9}$ 


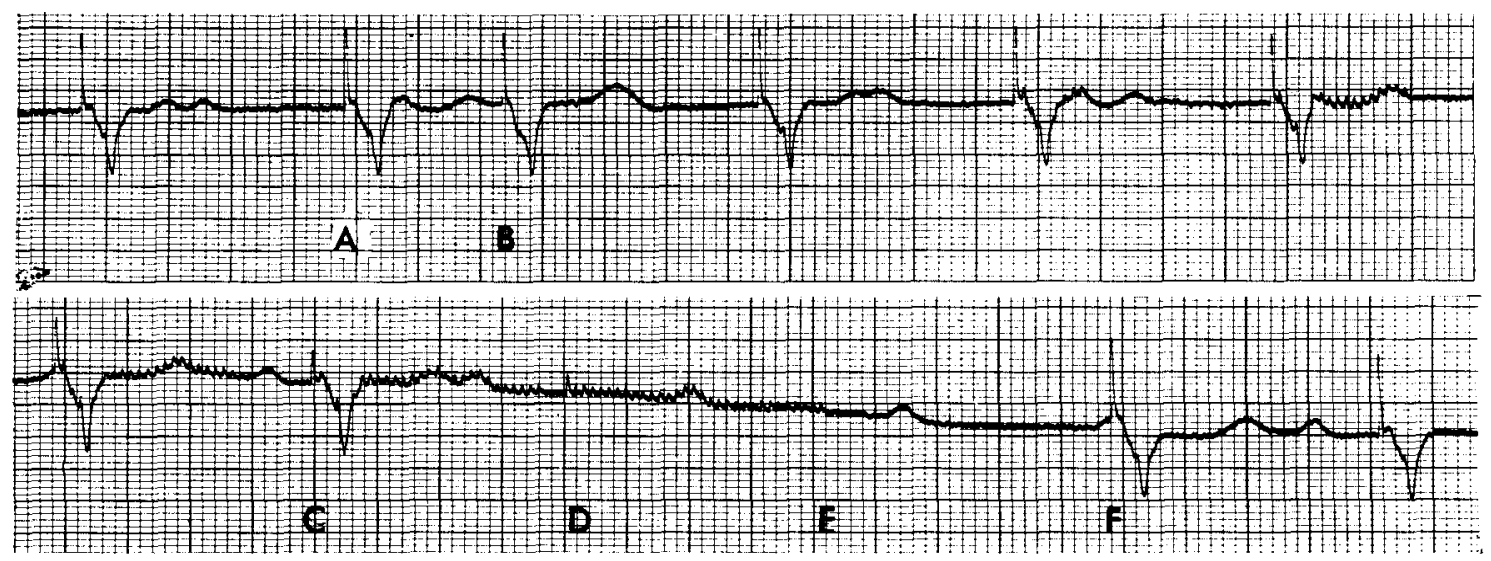

Fig. 2. Determination of threshold. A, normal electrical pacing. B, the threshold analyzer is turned on, capturing control of the implanted pacemaker. $\mathrm{C}$, between $\mathrm{B}$ and $\mathrm{C}$ the output of the implanted unit is slowly decreased, but the heart is still responding. $\mathrm{D}$, the stimulus has been reduced to a subthreshold level, with no myocardial response. The high-frequency artifacts are induced by the threshold analyzer. E, the threshold analyzer is turned off, allowing resumption of normal pacing. $F$, return of normal pacing.

Finally, all patients with exit block were given drug therapy in an attempt at restoring myocardial responsiveness. A wide variety of drugs and dosage levels were tried, but only two types will be included in this report: glucocorticoids and sympathomimetics.

\section{Results}

\section{STUDIES ON DOGS}

Studies on dogs showed that during the first week after implantation the threshold is lower than 2.5 per cent of the total pacemaker energy, or less than 1.45 microjoules. At this low threshold the dogs responded to the high frequency suppression pulses as well as the regular pace pulse, and thus experienced a pacemakerinduced tachycardia with a rate of around 200. This tachycardia is totally dependent on the threshold analyzer and can be terminated immediately by the operator. It was reproduced 20 times in 6 different dogs, with absolutely no sequellae after termination of the tachycardia by shutting off the threshold analyzer. In no case did ventricular fibrillation ensue, even when the tachycardia was allowed to persist for relatively long periods ( 15 to $30 \mathrm{sec}$.). In all 6 dogs the threshold level had risen to above 1.45 microjoules by 14 days postimplantation. In no human subject was such an arrhythmia produced, including one measurement taken 2 days following implantation. We do recommend, though, that routine measurements be deferred until the second postoperative week.

Serial measurements on 6 dogs showed that threshold energy increased sharply during the first two weeks and continued to increase up to three months postimplantation. Interelectrode impedance measured externally by methods previously reported showed a concomitant increase. Direct measurements on 3 of these animals showed that threshold is a function of total energy per pulse, not simply current.

Threshold as measured by this method in dogs and man has a very distinct end-point. Measurements are reproducable by different operators, and accurate to 1 per cent at threshold levels below 50 per cent, and to 2 per cent at levels above 50 per cent. As threshold with few exceptions remains below 50 per cent, the method is effectively accurate to 1 per cent.

\section{GLINIGAL STUDIES}

As a clinical tool, the threshold analyzer proved to be simple to operate, practical and safe. Total threshold analysis takes less than five minutes. In over 714 determinations on 28 human patients there were no arrhythmias, no significant symptoms during use, and no sequellae. Most patients reported a light-headed feeling if three or more consecutive heart beats were suppressed. For this reason, we make it a practice to restore pacing promptly after three stimuli have been missed. This is done by relaxing a control button on top of the coil which is held over the pacemaker, or by lifting the coil away from the pacemaker. It is necessary at all times that the operator of the threshold analyzer be watching a cardiac monitor so that he can immediately detect any suppressed beats.

Measurements on human subjects were made from two days to 30 months after implantation. Not one 


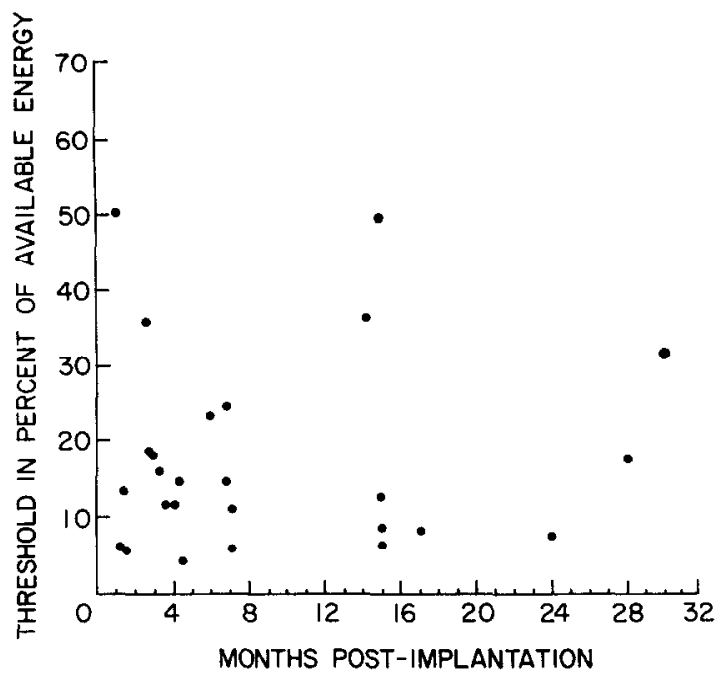

FIG. 3. Initial threshold measurements of 26 patients, taken from 1 to 30 months following implantation. Threshold energy is reported as a per cent of available pacemaker energy.

single extra heart beat was induced. The initial measurements of 26 patients are illustrated in Figure 3. Note that there is no apparent change in the distribution of threshold levels with time. In all cases except 2, threshold was less than 50 per cent of available energy and provided a safety factor of greater than 2:1. Serial measurements in 5 patients showed no significant change in threshold after three months of pacing. This also correlates with the previously reported finding that interelectrode resistance usually does not change significantly after three months. ${ }^{8}$

Threshold was clearly related to activity, rising significantly during sleep and falling after awakening. If a patient is kept awake but quiet, the threshold level changes less than 5 per cent in any hour. Exercise, on the other hand, can cause prompt reductions in threshold energy. These obscrvations have becn made repeatedly on several patients, but they have not yet been quantitated to our statisfaction.

Evaluation of 78 patients with exit block showed that this was the second most common cause of failure $(14 \%)$ in a series of 128 pacemaker implantations. Serum electrolytes were normal in all 18 cases. Sixteen ( $89 \%$ ) had had previous cardiac surgery. The onset in 16 cases occurred within two weeks following implantation, and in all cases the onset was within three nonths. Of the 18 patients, 14 were treated with steroids, and of these all but 2 returned to normal pacing within 72 hours, when treated with prednisone 40 to $60 \mathrm{mg}$. daily. The electrocardiogram in 1 of these cases is shown in Figure 4. Seven of these are still being paced on maintenance doses of 10 mg. daily. In 5, exit block conld not be prevented with acceptable maintenance doses of corticosteroids, and re-implantation was required. In 2 cases, exit block spontancously disappeared, and in 1 case the patient would not allow further treatment. In 15 of the cases threshold was grossly measured by reducing the pulse energy by means of an external rate control unit. ${ }^{9}$ Following treatment, threshold dropped to below 60 per cent of pacemaker energy in all but the 2 unresponsive cases. In 3 cases, exit block was anticipated before cessation of pacing. Serial measurements with the threshold analyzer demonstrated a rapidly rising threshold and interelectrode impendance in each instance. In all 3, clinical exit block developed. Trcatment with prednisone, $40 \mathrm{mg}$. daily, in 2 cases, and prednisone, $10 \mathrm{mg}$. plus ephedrine $25 \mathrm{mg}$. every six hours, in the third case, resulted in a fall of threshold below 33 per cent, giving a $3: 1$ safety factor. As the condition has improved in

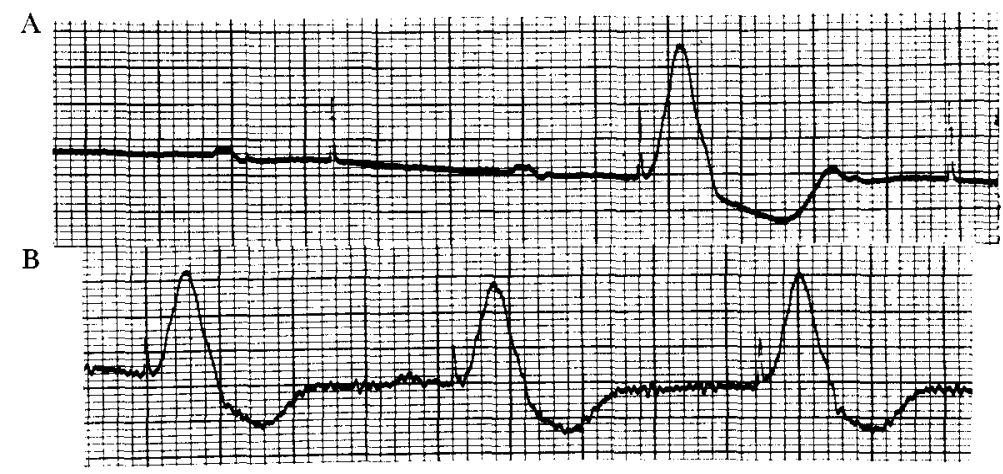

FIG. 1. Electrocardiogram of patient with exit block, (A) before and (B) 2.4 hours later after treatment with prednisone, $40 \mathrm{mg}$. Pacemaker rate $72 / \mathrm{min}$. 


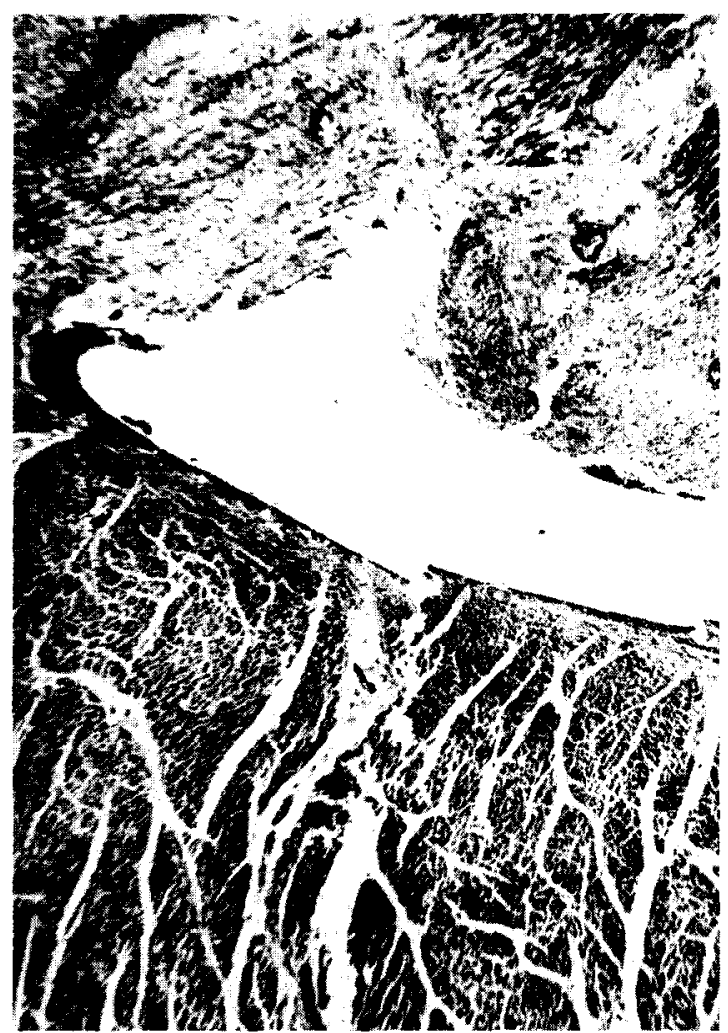

FIG. 5. Reaction around a myocardial electrode at 10 days following implantation (low power slide). The empty space is the clectrode tract. Notc minimal peri-electrode reaction. The patient died from a penicillin reaction.

each instance, we have gradually reduced the prednisone dosage, titrating it against a threshold level of 33 per cent or lower.

We have found that methylprednisolone and dexamethazone in equivalent doses can be substituted for prednisone without a significant difference in threshold effect. Preliminary observations do not support the substitution of hydrocortisone, as we have been unable to show a significant lowering of threshold with this drug. One patient treated with aldosterone, and another with 9-alpha fluorohydrocortisone, showed abrupt increases in threshold, suggesting a paradoxic effect from mineralocorticoids. Epinephrine, isoproterenol and ephedrine have all demonstrated the capacity to lower threshold in isolated instances.

\section{Discussion}

Knowledge of the threshold energy level with respect to pacemaker energy is essential to the diagnosis of exit block. The threshold analyzer, with which one can measure threshold energy as a per cent of available pacemaker energy,

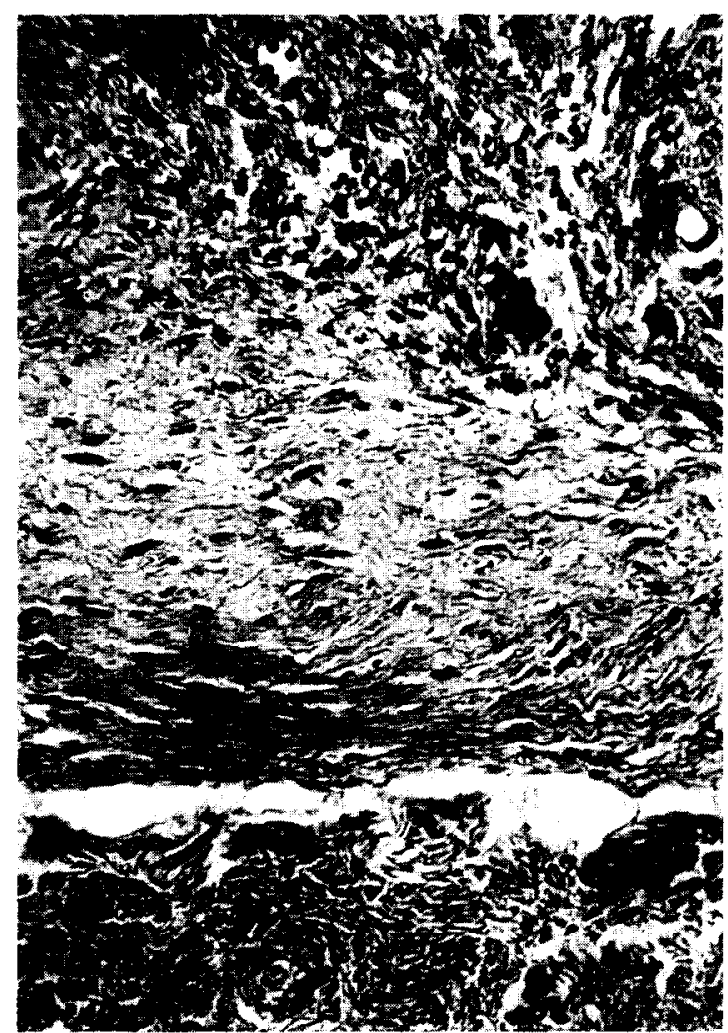

Fig. 6. Tissue reastion around the rlectrode tract at 6 months following implanlation (slide at same scale as Fig. 5). Note that the reaction is much greater than at 10 days. The patient died from noncardiac causes.

promises to be a simple and rational approach to the problem of detecting exit block before failure to pace develops. It also gives an analytical means of assessing the response to treatment.

Reaction Around Electrodes: Two important factors deserve consideration in exit block: reaction around the electrodes, and responsiveness of the myocardium. Figures 5 and 6 show perielectrode reaction in two different human patients who died from noncardiac causes. Note that the reaction at 10 days is minimal, whereas the reaction at six months is much greater. We have previously shown that the interelectrode impedance rises sharply during the first month postimplantation, but usually levels off by three months. Similarly, evidence to date in dogs and man suggests that the absolute threshold level follows the same pattern, rising during the first three months, after which there is little or no change. Equating interelectrode impedance and threshold measurements with tissue reaction around the electrodes gives a presumptive method of assessing inter- 


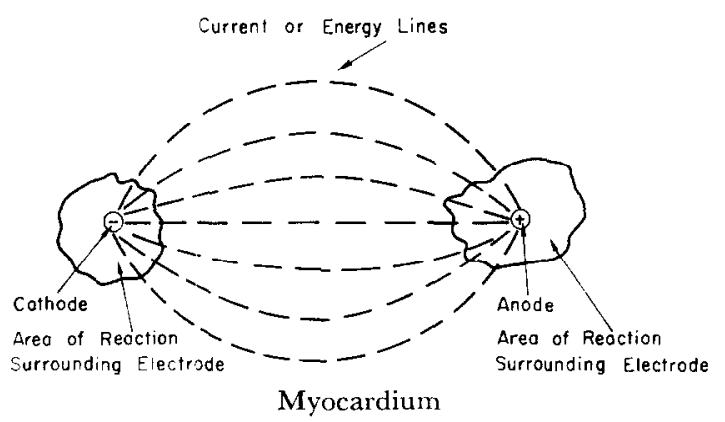

Hic. 7. Sichematic representation of peri-electrode reacion. Myocardial excitation takes place at the point of maximum energy density. As fibrosis increases around the clectrodes, the encrgy density at the point of cxcitation becomes less, thus raising the threshold energy requirement.

electrode tissue changes. Figure 7 is a scheinatic presentation of the perielectrode reaction. Myocardial excitation occurs where the energy density is greatest, and as seen in Figure 7, fibrosis around the electrodes results in nonresponsive tissue at the areas where the energy density is greatest. 7 Thus, the remaining normal myocardium between the electrodes is stimulated by a lower energy density, resulting in a higher energy threshold.

Responsireness of Myocardium: However, we found no consistent relation between absolute interelectrode impedance values and threshold. Threshold changes secondary to drugs or exercise were not accompanied by significant changes in interelectrode impedance. This observation tended to emphasize the relative importance of a second factor in myocardial electrical stimulation; i.e., the responsiveness of the myocardium itself. Transposing to a cellular level, threshold is dependent on two factors: the resting membrane potential, and the cellular threshold potential. Both are probably altered by sympathomimetic amines, and the importance of potassium on the resting membrane potential is well known. ${ }^{10-12}$ Theoretically, any agent which decreases the resting membrane potential, or increases the cellular threshold potential, will increase myocardial responsiveness. We were unable, however, to demonstrate hypo- or hyperkalemia in any of our patients with exit block.

Steroid Therapy for Exit Block: With the use of the threshold analyzer, the effect of drugs can be measured as definite changes in threshold energy. Steroid therapy for exit block is now based on clear-cut evidence that glucocorticoids decrease threshold energy requirements.
Mineralocorticoids, on the other hand, apparently increase threshold and, thercfore, should be avoided in exit block until their exact effect can be clarified by further study. Although prednisone reduces threshold, there is no evidence as yet that it reduces ultimate perielectrode reaction, and therefore we do not use it routinely after implantation. Although our information to date is less complete, there is no question that sympathomimetic anines also have the capacity to reduce threshold energy requirements significantly.

Our criterion for treatment to increase myocardial responsiveness in impending exit block is now a threshold level which rises above 50 per cent of the pacemaker energy within the first three months. Moreover, if threshold reaches 10 per cent within the first weck after implantation, 25 per cent in the first two weeks, or 50 per cent in the first month, exit block is very likely unless prevented by treatment. In such cases we recommend treatment initially with $40 \mathrm{mg}$. of prednisone per day, until the threshold either levels off or falls to below 33 per cent ( $3: 1$ safety factor). At this point the prednisone should be reduced to the lowest possible dosage which will sustain threshold below 33 per cent. Initially, threshold measurements are taken every day. After stabilization of threshold, measurements are reduced to cvery two to four weeks.

Baltery Failure: Another clinical use of the threshold analyzer is in battery failure. When the batteries are almost completely depleted, there is usually a gradual decline of battery voltage from normal to subthreshold levels. With the pacemaker used in this study, the diagnosis of impending battery failure is easily made, ${ }^{8}$ but to date there has been no means of determining the magnitude of the decrease in voltage or its significance with respect to myocardial stimulation. If the battery voltage level has dropped dangerously low, emergency surgical replacement is mandatory to prevent the pacemaker energy output from falling below the myocardial threshold. However, if the battery failure is detected early, and the ratio between threshold energy and pacemaker output energy can be demonstrated not to be changing quickly, surgical replacement of the unit can be done under more optimal nonemergency conditions. Such analysis also obviates placement of a temporary catheter pacenaker. All evidence to date is that threshold energy levels do not change progressively after three 
months following myocardial electrode implantation. Since threshold energy is measured as a per cent of pacemaker energy after three months, it is reasonable to assume in known battery failure that an increase in relative threshold is due to a decrease in pacemaker energy, and not an incrcasc in absolutc threshold cnergy requirement.

The results show that in many cases very low threshold levels persist after the three month postimplantation period. Threshold changes due to fibrosis occur only after implantation of new myocardial electrodes, not after replacement of a new power unit on the old wires. We therefore believe that if a persistently low threshold can be demonstrated, when battery failure ultinately occurs the power unit can be replaced by a low-energy unit, still ensuring at least a 3:1 safety factor but taking advantage of the low threshold level to economize on battery usage. It is anticipated that by safely reducing pacemaker output energy in such patients, the battery life may be extended significantly, and any potentially harmful effects from excessive current flow between the electrodes also may be minimized.

All the above methods apply to only one pacemaker, but they should be valid whether it is attached to myocardial electrodes or to a transvenous catheter electrode. The potential value of threshold analysis in pharmacologic research is self-evident.

\section{SUMmary}

A method for estimating myocardial threshold in patients with implanted artificial pacemakers has been described. By the external application of a calibrated suppression signal, the output of the implanted unit can be gradually reduced until the myocardial response ceases. The threshold level thus determined can be expressed as a per cent of available pulse energy by a simple calculation.

This method promises to have clinical application because it is relatively simple and entirely external. It has been demonstrated to be safe, no sequelae having occurred during over 700 human studies. In combination with our previously reported method of estimating interelectrode impedance, it should provide a rational means of serially evaluating pacemaker function following implantation. It is applicable to both direct myocardial and transvenous modes of pacing, but its most important limitation at the present time is that it can be used with only one of the commercially available pacemakers.

By application of quantitative threshold measurements, we have been able to confirm our clinical observations of exit block as a significant cause of pacemaker failure. This complication compriscd the second most common cause of failure to pace, 14 per cent in a series of 128 implantations. It has also been possible by this means to verify the effectiveness of glucocorticoid and sympathomimetic drugs in lowering the myocardial threshold level. The implications of these observations have been briefly discussed.

Threshold analysis may prove to be an important means of investigating the myocardial effects of other pharmacologic preparations in the future.

\section{REFERENCES}

1. Parsonnet, V., Gilbert, L., Zucker, R. and Asu, M. M. Complications of the implanted pacemaker. J. Thoracic \& Cardiovas. Surg., 45:801, 1963.

2. Wallace, H. W., Wisoff, B. G., Gadboys, H. L. and LiTwak, R. S. Management of malfunctioning implanted cardiac pacemakers. Circulation, 32 (Suppl. 2): 214, 1965.

3. Harris, A., et al. The management of heart block. Brit. Heart J., $27: 469,1965$.

4. Chardack, W. M. Heart block treated with an implantable pacemaker: Past experience and current developments. Prog. Cardiovas. Dis., 6: $507,1964$.

5. Zoll, P. M., Frank, H. A. and Linenthal, A. J. Four year experience with an implanted cardiac pacemaker. Ann. Surg., 160: 351, 1964.

6. Townsend, J. F., Stoeckle, H. and Schuder, J. C. Tissue and electrode changes in chronic cardiac pacing: An experimental study. Tr. Am. Soc. Arif. Int. Organs, 11: 132, 1965.

7. Mansfield, P.B. and Cole, A. D. The design and analysis of myocardial electrodes. Proceedings of 16th Annual Conference on Engineering in Medicine and Biology, Baltimore, Md., Nov. 18-20, 1963.

8. Preston, T. A., Judge, R. D., Bowers, D. L. and Morris, J. D. Measurement of pacemaker performance. Am. Heart $J ., 71: 92,1966$.

9. Kantrowitz, $A$. The treatment of Stokes-Adams syndrome in heart block. Prog. Cardiozas. Dis., 6: $490,1964$.

10. Hoffman, B. F. and Cranefield, P. F. Electrophysiology of the Heart, p. 91. New York, 1960. McGraw-I Iill Book Co., Inc.

11. Reynolds, E. W. Ionic transfer in cardiac muscle. An explanation of cardiac electrical activity. $\mathrm{Am}$. Heart J., 67: 693, 1964.

12. Walker, W. J., Elkins, J. T. and Wood, L. W. Effect of potassium in restoring myocardial response to a subthreshold cardiac pacemaker. New England J. Med., 271: 597, 1964. 